\title{
Terahertz Raman laser based on silicon doped with phosphorus
}

\author{
S. G. Pavlov, ${ }^{1, a)}$ H.-W. Hübers, ${ }^{1}$ U. Böttger, ${ }^{1}$ R. Kh. Zhukavin, ${ }^{2}$ V. N. Shastin, ${ }^{2}$ \\ J. N. Hovenier, ${ }^{3}$ B. Redlich, ${ }^{4}$ N. V. Abrosimov, ${ }^{5}$ and H. Riemann ${ }^{5}$ \\ ${ }_{1}^{1}$ Institute of Planetary Research, German Aerospace Center (DLR), Rutherfordstraße 2, 12489 Berlin, \\ Germany \\ ${ }^{2}$ Institute for Physics of Microstructures, Russian Academy of Sciences, 603950 Nizhny Novgorod, Russia \\ ${ }^{3}$ Kavli Institute of Nanoscience Delft, Delft University of Technology, 2600 GA Delft, The \\ Netherlands \\ ${ }_{5}^{4}$ FOM-Institute for Plasma Physics, 3439 MN Nieuwegein, The Netherlands \\ ${ }^{5}$ Institute of Crystal Growth, Max-Born-Straße 2, 12489 Berlin, Germany
}

(Received 23 November 2007; accepted 13 February 2008; published online 4 March 2008)

\begin{abstract}
Raman-type stimulated emission at frequencies between 5.0 and $5.2 \mathrm{THz}$ as well as between 6.1 and 6.4 THz has been realized in silicon crystals doped by phosphorus donors. The Raman laser operates at around $5 \mathrm{~K}$ under optical excitation by a pulsed, frequency-tunable infrared free electron laser. The frequencies of the observed laser emission are close to the frequencies of the intracenter laser lines which originate from the $2 p_{0}$ and $2 p_{ \pm}$phosphorus states. The Stokes shift of $3.16 \mathrm{THz}$ is equal to the difference between the energies of the phosphorus ground state, $1 s\left(A_{1}\right)$, and the $1 s(E)$ excited state. (C) 2008 American Institute of Physics. [DOI: 10.1063/1.2890717]
\end{abstract}

Over the past years, a lot of effort has been devoted to the development of silicon-based lasers. These efforts were driven by the need for low cost photonic devices which are fully integrable into optoelectronic circuits. The main difficulty in realizing silicon lasers is its indirect energy gap between the conduction and valence bands that forbids direct optical recombination of a free electron. Some major approaches to overcome the inefficient luminescence in silicon rely on the enhancement of the efficiency of bandgap transitions by quantum confinement effects in low dimensional silicon-based materials ${ }^{1-3}$ and band structure engineering in heterostructures. ${ }^{4-6}$ So far, none of these approaches has led to stimulated emission. The first silicon laser has been demonstrated on direct optical transitions between particular energy levels of group- $\mathrm{V}$ donors in silicon. In this type of laser, population inversion can be achieved due to the peculiarities of the interaction of the phonons in silicon with the donor electrons. ${ }^{7,8}$ The lasers are optically pumped at infrared wavelengths and emit at terahertz frequencies.

Raman-type lasers based on conversion of infrared light in the lattice of silicon have been demonstrated recently. ${ }^{9,10}$ In these lasers, the strongest Stokes emission is determined by scattering on the threefold degenerate short-wavelength optical modes at the center of the Brillouin zone of silicon (phonon energy $64.35 \mathrm{meV} \approx 15.53 \mathrm{THz}$ ). A terahertz Raman-type all-intracenter silicon laser doped by antimony $(\mathrm{Si}: \mathrm{Sb})$ has been reported in $2006 .{ }^{11}$ In the intracenter Raman $\mathrm{Si}: \mathrm{Sb}$ laser scattering of pump photons occurs on two lower donor states, resonantly coupled via the intervalley transverse acoustic (TA) $g$-phonon [phonon energy $\hbar \omega_{g \text {-TA }}$ is $11.4 \mathrm{meV}$ for pure silicon at a lattice temperature of $65 \mathrm{~K}$ (Ref. 12)]. This interaction significantly enhances the scattering efficiency and Raman gain. Here, we report on the realization of a terahertz Raman laser made from silicon doped by phosphorus.

The silicon crystals used in our experiments were grown by the float zone technique in the [111] direction. Phosphorus

\footnotetext{
a) Author to whom correspondence should be addressed. Tel.: +493067055594. FAX: +493067055507. Electronic mail: sergeij.pavlov@dlr.de.
}

donors were incorporated by simultaneous doping from the melt. The donor concentration was $N_{D}=(2-3) \times 10^{15} \mathrm{~cm}^{-3}$ and the compensation was $N_{A} / N_{D}<0.001$, where $N_{A}$ is the residual acceptor concentration in the crystal. The investigated samples were rectangular parallelepipeds with dimensions of about $7 \times 7 \times 5 \mathrm{~mm}^{3}$. The largest facet was orthogonal to the growth axis. All facets were chemically optically polished parallel to each other with an accuracy of 1 arc min. This forms a low-loss resonator for modes on total internal reflection. We used the free electron laser (FEL) FELIX at the IR-User facility of the FOM Institute for Plasma Physics, Rijnhuizen, The Netherlands, for pumping the silicon lasers. For these experiments, FELIX generated $\sim 6 \mu$ s long macropulses at a $5 \mathrm{~Hz}$ repetition rate in the range from 27 to $40 \mu \mathrm{m}(7.7-11.1 \mathrm{THz}, 32-46 \mathrm{meV})$. Each macropulse consisted of a train of micropulses with a duration of about $6-8$ ps separated by 1 ns. The FEL power incident on the sample was controlled by a calibrated step attenuator and measured by a Joule meter (Molectron Energy Max 500). The spectrum of the pump pulse of the FEL had a Lorentzian shape with a full width at half maximum of about $0.1-0.2 \mathrm{meV}$. The accuracy of the central wavelength was $\sim 0.04 \mathrm{meV}$. The silicon samples were mounted in a holder, which was immersed into a liquid helium $(1 \mathrm{He})$ transport vessel providing a temperature of $4.2 \mathrm{~K}$. The pump radiation was guided to the $\langle 111\rangle$ facet of the sample by a stainless steel tube. A thin polyethylene (PE) film on top of the pump tube served as a window for the pump radiation. For measurements of the laser threshold, the terahertz emission was detected by a Ge:Ga detector orthogonal to the pump radiation. A sapphire filter and $\mathrm{a} \mathrm{CaF}_{2}$ filter, which were placed between the sample and the detector, blocked the pump radiation (inset in Fig. 1), which might have been scattered or reflected in direction of the detector. For spectral measurements, the terahertz emission was directed out of the transport vessel by a $45^{\circ}$ mirror and another stainless steel tube and focused onto a $1 \mathrm{He}$ cooled Ge:Ga photoconductive detector sensitive at wavelengths shorter than $140 \mu \mathrm{m}$ $(>8.8 \mathrm{meV})$. Any remaining radiation from the FEL was blocked by a $1 \mathrm{~mm}$ thick $z$-cut crystalline quartz filter and a high density PE filter. The emission spectra (Fig. 1) were 


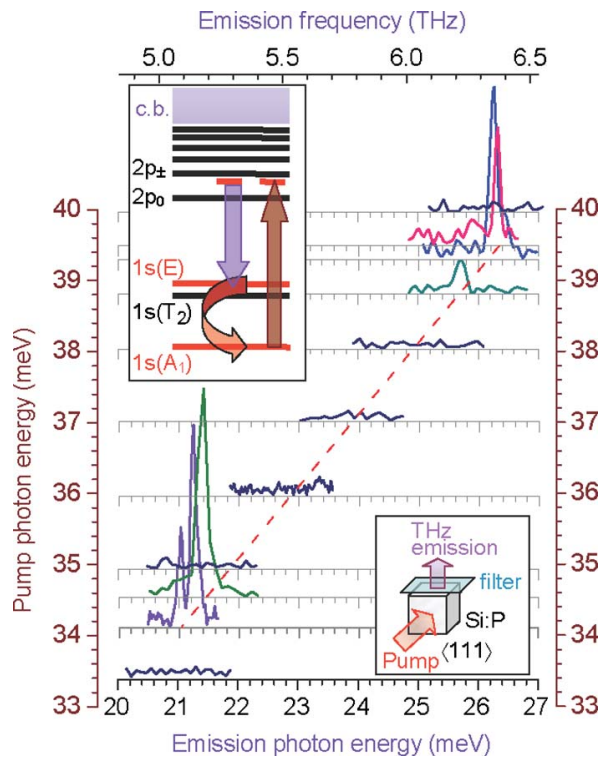

FIG. 1. (Color online) Raman-type emission spectra of a Si:P laser obtained at maximum pump power. The vertical axis shows the pump photon energy (baseline of each emission spectrum). The intensity is plotted linearly. The lower inset shows a sketch of the experimental setup. The upper inset shows the scheme of the Raman laser process: pumping occurs from the ground state $1 s\left(A_{1}\right)$ into a virtual state (dashed line). An excited electron decays in the $1 s(E)$ donor state with the emission of a terahertz photon and finally relaxes nonradiatively by emission of an intervalley $g$-TA phonon.

measured with a Fourier transform spectrometer (FTS) (resolution $\sim 0.05-0.06 \mathrm{meV}, \sim 0.4-0.5 \mathrm{~cm}^{-1}$ ) for pump frequencies in the range from 32 to $46 \mathrm{meV}(7.7-11.1 \mathrm{THz})$ (Fig. 2). This covers excitation from the donor ground state, $1 s\left(A_{1}\right)$, into all excited states as well as into the conduction band (c.b.) continuum (inset in Fig. 1).
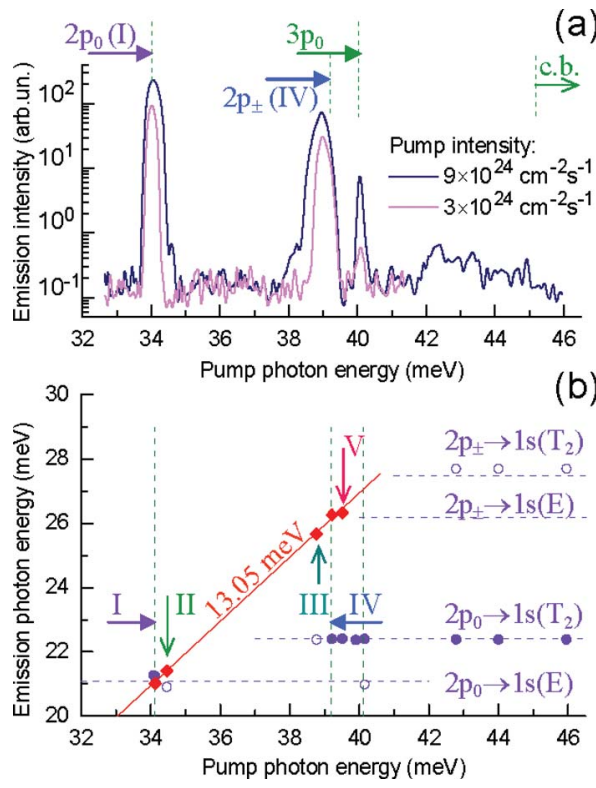

FIG. 2. (Color online) Dependences of the emission intensity (a) and emission photon energy (b) on the pump photon energy. The arrows in (a) as well as the vertical lines in (b) indicate the positions of the photon energies which correspond to resonant pumping in excited donor states. The filled symbols in (b) indicate strong lines and the open cycles are for the weak ones. Diamonds show the lines corresponding to the Stokes shifted Raman emission. The solid line in (b) shows the linear fit for the frequency shift of the Stokes emission. The horizontal dashed lines in (b) indicate the Si:P donor laser transition. Labels I to V indicate pump energy for the Raman emission

lines.
Downloaded 09 Aug 2010 to 131.180.130.114. Redistribution subject to AIP license or copyright; see http://apl.aip.org/apl/copyright.jsp

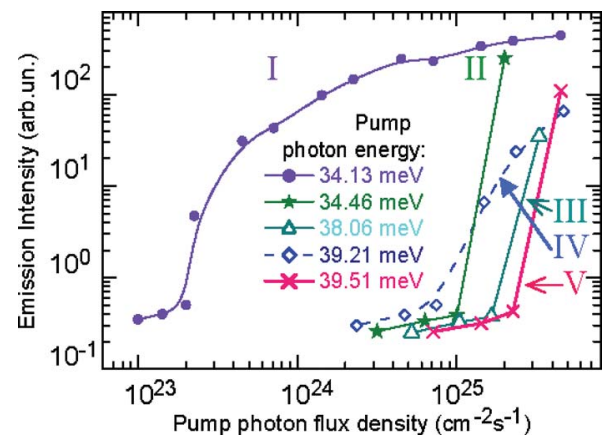

FIG. 3. (Color online) Typical dependences of the terahertz emission from Si:P on the pump photon flux density.

It has been shown that intracenter pumping of $\mathrm{Si}: \mathrm{P}$ with moderate optical pump power (i.e., below about $10^{24}$ photons $\mathrm{cm}^{-2} \mathrm{~s}^{-1}$ ) results in stimulated emission occurring each time when the pump photon energy corresponds to a dipole allowed optical transition originating from the ground state, $1 s\left(A_{1}\right) .{ }^{13}$ Lasing occurs exclusively on two donor transitions. ${ }^{14}$ These are the $2 p_{0} \rightarrow 1 s(E)$ transition, when pumped resonantly in the $2 p_{0}$ phosphorus state, and the $2 p_{0} \rightarrow 1 s\left(T_{2}\right)$ donor transition, when pumped in any other donor state above $2 p_{0}$ and up to the conduction band. ${ }^{8}$ At maximum pump power available from FELIX (about $10^{25}$ photons $\mathrm{cm}^{-2} \mathrm{~s}^{-1}$ ) a major change happens (Fig. 2). Additional emission lines appear even when the pump frequency does not correspond to an intracenter transition but is close to the two low energy donor transitions $1 s\left(A_{1}\right) \rightarrow 2 p_{0}$ $(34.11 \mathrm{meV})$ and $1 s\left(A_{1}\right) \rightarrow 2 p_{ \pm}(39.19 \mathrm{meV})$ (Fig. 2). Its photon energy $\hbar \omega_{S}$ varies with the pump photon energy $\hbar \omega_{\mathrm{FEL}}$ as [Figs. 1 and 2(b)]

$$
\hbar \omega_{S}=\hbar \omega_{\mathrm{FEL}}-\Delta E,
$$

where $\Delta E=13.05 \pm 0.05 \mathrm{meV} \quad(3.16 \pm 0.01 \mathrm{THz})$ is very close to the interstate energy gap between the phosphorus ground state and the split-off doublet $1 s(E)$, which is $13.01 \mathrm{meV}(3.14 \mathrm{THz}) .{ }^{15}$ We suppose that except for one line (one of the two lines which occur at pump energy of $34.1 \mathrm{meV}$, marked with I in Fig. 2) all the others are due to stimulated, Stokes-shifted Raman emission. The stimulated emission corresponding to lines number II and III has an almost two order of magnitude larger laser threshold (Fig. 3) than those laser lines originating from resonant intracenter pumping transitions. In addition, they are significantly offset from any intracenter pump transition. Two lines (marked by IV and V in Fig. 2) have a frequency close to the $2 p_{ \pm} \rightarrow 1 s(E)$ transition. They have as well large laser thresholds. Since the optical cross section of the $2 p_{ \pm} \rightarrow 1 s(E)$ transition is smaller than that of the $2 p_{ \pm} \rightarrow 1 s\left(T_{2}\right)$ transition, the lines are not present in the emission spectra of Si:P lasers pumped with moderate power. ${ }^{14}$ In addition, one of the lines appears at a pump frequency which is significantly offset from resonant excitation into the $2 p_{ \pm}$state. Therefore, we assume that both lines are due to Raman emission. In the case of resonant pumping into the $2 p_{0}$ state [marked by I in Fig. 2(b)], the emission frequency might be described by Eq. (1). However, the frequency difference to the intracenter $2 p_{0} \rightarrow 1 s(E)$ transition is not significant. The temporal structure and the spectrum of the emission pulse at this pump frequency are shown in Fig. 4. The pulse has two maxima at the maximum pump power of $5 \times 10^{25}$ photons $\mathrm{cm}^{-2} \mathrm{~s}^{-1}$. The AIP license or copyright; see http://apl.ailp.org/apl/copyright.jsp 


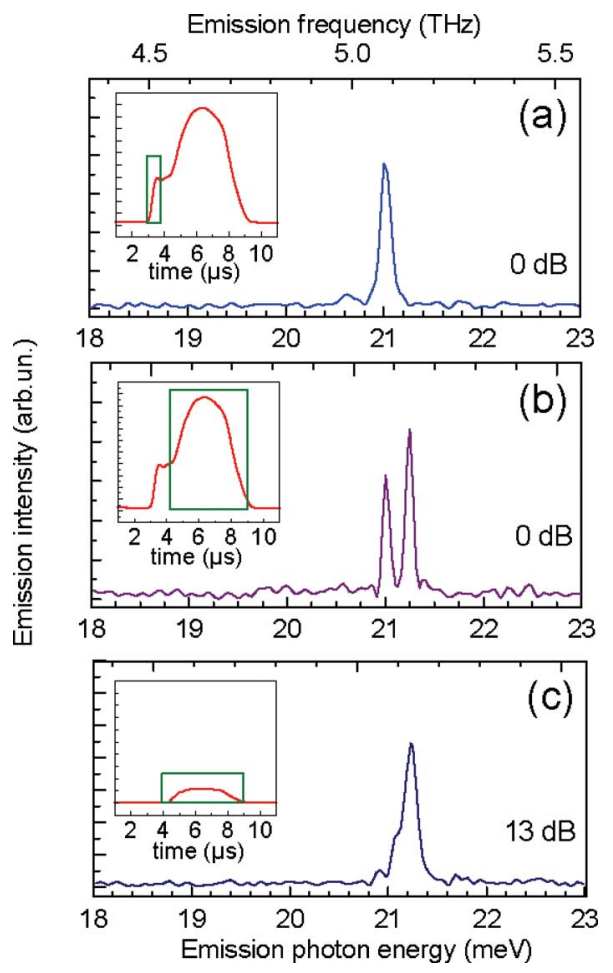

FIG. 4. (Color online) Emission spectra and corresponding emission pulses (insets) recorded for the case of resonant pumping into the $2 p_{0}$ state (line I in Figs. 2 and 3). The spectra are taken with different time gates shown by the rectangles in the insets. The labels in $\mathrm{dB}$ indicate the attenuation of the pump power; $0 \mathrm{~dB}$ corresponds to $2 \times 10^{25}$ photons $\mathrm{cm}^{-2} \mathrm{~s}^{-1}$.

relative intensity in the maxima is rather unstable from one pump pulse to the next indicating a competing character. When decreasing the pump power the front peak disappears and the pulse becomes stable. Spectral measurements performed with a time gate (shown by rectangles in Fig. 4) show that the front part of the emission pulse corresponds to one strong line at $21.00 \mathrm{meV}$. It does not correspond to an intracenter transition. This line [Fig. 4(a)] has a significantly $(\sim 15 \mathrm{~dB})$ higher laser threshold and appears first after the pump pulse. The second part of the emission pulse appears with a $\sim 0.5-1 \mu$ s delay. The spectrum corresponding to this part of the pulse consists of two lines. One is the line which is attributed to the first pulse peak which signal probably persists. The frequency of the other line corresponds to the $2 p_{0} \rightarrow 1 s\left(T_{2}\right)$ transition $(21.25 \mathrm{meV})$. The front peak of the emission pulse and the corresponding line in the emission spectrum disappear at pump intensities below $10^{25} \mathrm{~cm}^{-2} \mathrm{~s}^{-1}$ [Fig. 4(c)]. For these reasons, we attribute the low frequency line to Raman emission while the high frequency line is due to the intracenter $2 p_{0} \rightarrow 1 s(E)$ transition. It is worth noting that the peak power of the Raman emission pulse was estimated to be a few milliwatts at maximum pump power. There are other lines in the emission spectra whose frequency does not change with pump power. These lines can be attributed to the intracenter transitions $2 p_{ \pm} \rightarrow 1 s\left(T_{2}\right)$ and $2 p_{0} \rightarrow 1 s\left(T_{2}\right)$. The emission from the $2 p_{ \pm} \rightarrow 1 s\left(T_{2}\right)$ transition is rather weak due to the competition with the $2 p_{0} \rightarrow 1 s\left(T_{2}\right)$ which is more efficiently populated when pumping occurs into higher excited states or into the conduction band. ${ }^{8,14}$

Although the Raman-type emission in Si:P has similarity to that observed in Si:Sb media, ${ }^{11}$ it has some new features. The major difference is that the Raman laser effect in Si:P has been observed only in close vicinity to the $2 p_{0}$ and $2 p_{ \pm}$ states at frequencies of 5.0-5.2 and 6.1-6.4 THz, where the condition of the "incoming" Raman resonance is almost satisfied. ${ }^{16}$ Contrary, in Si:Sb Raman lasers, emission has been also observed for pumping far off from any intracenter electronic state. We attribute this difference to the absence of the resonant coupling of the $1 s\left(A_{1}\right)$ and $1 s(E)$ states with the intervalley phonon ("outgoing" Raman resonance ${ }^{15}$ ), as it occurs in the $\mathrm{Si}: \mathrm{Sb}$ laser. This causes a significant decrease of the Raman gain over the entire frequency range. As a result, Raman lasing in Si:P requires relatively high pump power and does not cover the entire energy range between the $2 p_{0}$ and $2 p_{ \pm}$states as in the case of the Si:Sb Raman laser.

In summary, we have observed Raman-type stimulated Stokes emission in silicon crystals doped by phosphorus. The Stokes shift is determined by the phosphorus electronic transition between $1 s\left(A_{1}\right)$ and $1 s(E)$ states. The lowest laser threshold and highest output power have been obtained for the degenerated case of pumping into the $2 p_{0}$ state when intracenter laser emission and Stokes emission occur simultaneously. The different performance of the Si:P Raman laser compared to the Si:Sb Raman laser is attributed to the absence of the outgoing Raman resonance between the impurity states and the $g$-TA phonon.

This work was partly supported by the European Commission through the ProFIT programme of the Investitionsbank Berlin and the Deutsche Forschungsgemeinschaft. We acknowledge the support by the Stichting voor Fundamenteel Onderzoek der Materie (FOM) in providing the required beam time on FELIX and appreciate the assistance by N. Q. Vinh and R. van Buuren.

${ }^{1}$ L. Pavesi, Mater. Today 1, 18 (2005).

${ }^{2}$ M. J. Chen, J. L. Yen, J. Y. Li, J. F. Chang, S. C. Tsai, and C. S. Tsai, Appl. Phys. Lett. 84, 2163 (2004).

${ }^{3}$ S. G. Clouter, P. A. Kossyrev, and J. Xu, Nat. Mater. 4, 887 (2005).

${ }^{4}$ Z. H. Lu, D. J. Lockwood, and J. M. Baribeau, Nature (London) 378, 258 (1995).

${ }^{5}$ G. Dehlinger, L. Diehl, U. Gennser, H. Sigg, J. Faist, K. Ensslin, D. Grützmacher, and E. Müller, Science 290, 2277 (2000).

${ }^{6}$ J. Zhang, X. B. Li, J. H. Neave, D. J. Norris, A. G. Cullis, R. W. Kelsall, S. Lynch, P. Towsend, D. J. Paul, and P. F. Fewster, J. Cryst. Growth 278, 488 (2005)

${ }^{7}$ S. G. Pavlov, R. Kh. Zhukavin, E. E. Orlova, V. N. Shastin, A. V. Kirsanov, H.-W. Hübers, K. Auen, and H. Riemann, Phys. Rev. Lett. 22, $5220(2000)$.

${ }^{8}$ H.-W. Hübers, S. G. Pavlov, and V. N. Shastin, Semicond. Sci. Technol. 20, S211 (2005).

${ }^{9}$ O. Boyaraz and B. Jalali, Opt. Express 12, 5269 (2004).

${ }^{10}$ H. Rong, A. Liu, R. Jones, O. Cohen, D. Hak, R. Nicolaescu, A. Fang, and M. Paniccia, Nature (London) 433, 292 (2005); H. Rong, R. Jones, A. Liu, O. Cohen, D. Hak, A. Fang, and M. Paniccia, ibid. 433, 725 (2005).

${ }^{11}$ S. G. Pavlov, H.-W. Hübers, J. N. Hovenier, T. O. Klaassen, D. A. Carder, P. J. Phillips, B. Redlich, H. Riemann, R. Kh. Zhukavin, and V. N. Shastin, Phys. Rev. Lett. 96, 037404 (2006).

${ }^{12}$ M. Asche and O. G. Sarbei, Phys. Status Solidi B 103, 11 (1981), and references therein.

${ }^{13}$ V. N. Shastin, R. Kh. Zhukavin, E. E. Orlova, S. G. Pavlov, M. H. Rümmeli, H.-W. Hübers, N. Hovenier, T. Klaassen, H. Riemann, I. V. Bradley, and A. F. G. van der Meer, Appl. Phys. Lett. 80, 3512 (2002).

${ }^{14}$ R. Kh. Zhukavin, D. M. Gaponova, A. V. Muravjov, E. E. Orlova, V. N. Shastin, S. G. Pavlov, H.-W. Hübers, J. N. Hovenier, T. O. Klaassen, H. Riemann, and A. F. G. van der Meer, Appl. Phys. B: Lasers Opt. 76, 613 (2003).

${ }^{15}$ A. K. Ramdas and S. Rodriguez, Rep. Prog. Phys. 44, 1297 (1981).

${ }^{16} \mathrm{P}$. Y. Yu and M. Cardona, Fundamentals of Semiconductors, 3rd ed. (Springer, Berlin, 2005), p. 394. 